\title{
Mixed Convection Magnetohydrodynamic (MHD) Flow of Walters-B Liquid Fluid past an Accelerating Permeable Surface with Soret-Dufour Mechanism
}

\author{
Bidemi Falodun ${ }^{1}$ and Cletus Onwubuoya ${ }^{1}$ \\ ${ }^{1}$ University of Ilorin
}

May 6, 2020

\begin{abstract}
This study deals with the MHD convective flow of Walters-B memory viscoelastic fluid over an accelerating permeable surface under the influence of Soret and Dufour. The modeled equations are set of partial differential equations. These equations are transformed into coupled nonlinear ordinary differential equations with the use of suitable similarity transformation. The thermal radiative heat flux is modeled using the Roseland approximation. Hence, the fluid considered in this study is optically thin. The numerical solution was profound to the transformed equations using spectral homotopy analysis method (SHAM) and results are presented in graphs and tables. The influence of Soret and Dufour are investigated separately. Results hereby revealed that the effect of Soret and Dufour on temperature and concentration profiles is the opposite.
\end{abstract}

\section{Hosted file}

EditedManuscript.pdf available at https://authorea.com/users/317321/articles/448812-mixedconvection-magnetohydrodynamic-mhd-flow-of-walters-b-liquid-fluid-past-an-acceleratingpermeable-surface-with-soret-dufour-mechanism 


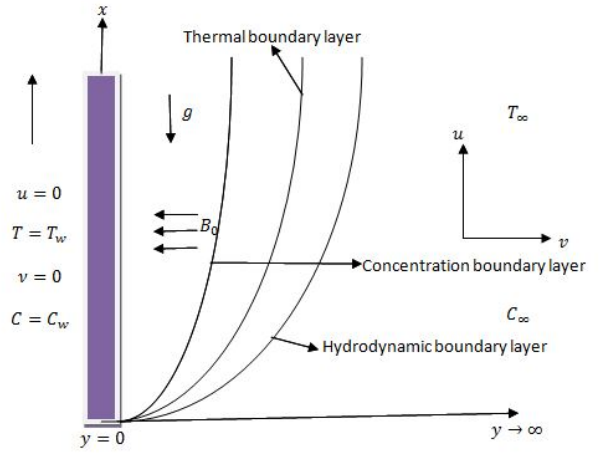

Figure 1. Flow configurations

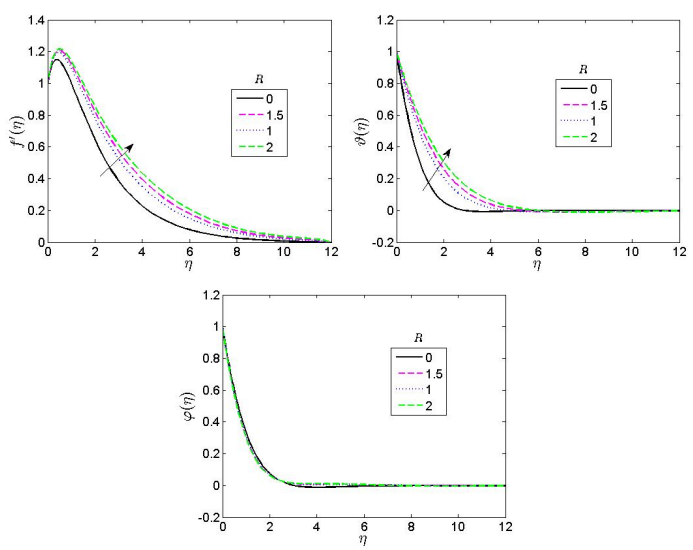

Figure 2: Effect of $R$ on the velocity, temperature and concentration profiles 
figures/alphaconc/alphaconc-eps-converted-to.pdf 
figures/alphatemp/alphatemp-eps-converted-to.pdf 
figures/alphavel/alphavel-eps-converted-to.pdf 
figures/betaconc/betaconc-eps-converted-to.pdf 
figures/betatemp/betatemp-eps-converted-to.pdf 
figures/betavel/betavel-eps-converted-to.pdf 
figures/Df conc/Df conc-eps-converted-to.pdf 
figures/Dftemp/Dftemp-eps-converted-to.pdf 
figures/Dfvel/Dfvel-eps-converted-to.pdf 
figures/Econc/Econc-eps-converted-to.pdf 
figures/Etemp/Etemp-eps-converted-to.pdf 
figures/Evel/Evel-eps-converted-to.pdf 
figures/Gm/Gm-eps-converted-to.pdf 
figures/Gmconc/Gmconc-eps-converted-to.pdf 
figures/Gmtemp/Gmtemp-eps-converted-to.pdf 
figures/Gtconc/Gtconc-eps-converted-to.pdf 
figures/Gttemp/Gttemp-eps-converted-to.pdf 
figures/Gtvel/Gtvel-eps-converted-to.pdf 
figures/Mconc/Mconc-eps-converted-to.pdf 
figures/Mtemp/Mtemp-eps-converted-to.pdf 
figures/Mvel/Mvel-eps-converted-to.pdf 
figures/Rconc/Rconc-eps-converted-to.pdf 
figures/Rtemp/Rtemp-eps-converted-to.pdf 
figures/Rvel/Rvel-eps-converted-to.pdf 
figures/Prconc/Prconc-eps-converted-to.pdf 
figures/Prtemp/Prtemp-eps-converted-to.pdf 
figures/Prvel/Prvel-eps-converted-to.pdf 
figures/Sc/Sc-eps-converted-to.pdf 
figures/Scconc/Scconc-eps-converted-to.pdf 
figures/Sctemp/Sctemp-eps-converted-to.pdf 
figures/Swconc/Swconc-eps-converted-to.pdf 
figures/Swtemp/Swtemp-eps-converted-to.pdf 
figures/Swvel/Swvel-eps-converted-to.pdf 
figures/Srconc/Srconc-eps-converted-to.pdf 
figures/Srtemp/Srtemp-eps-converted-to.pdf 
figures/Srvel/Srvel-eps-converted-to.pdf 